\title{
Protein function prediction with gene ontology: from traditional to deep learning models
}

Vu Thi Thuy Duong ${ }^{1}$, Jaehee Jung ${ }^{\text {Corresp. } 1}$

${ }^{1}$ Department of Information and Communication Engineering,, Myongji University, Gyeonggi-do, Gyeonggi-do, South Korea

Corresponding Author: Jaehee Jung

Email address: jhjung@mju.ac.kr

Protein function prediction is a crucial part of genome annotation. Prediction methods have recently witnessed rapid development, owing to the emergence of high-throughput sequencing technologies. Among the available databases for identifying protein function terms, Gene Ontology (GO) is an important resource that describes the functional properties of proteins. Researchers are employing various approaches to efficiently predict the GO terms. Meanwhile, deep learning, a fast-evolving discipline in data-driven approach, exhibits impressive potential with respect to assigning GO terms to amino acid sequences. Herein, we reviewed the currently available computational GO annotation methods for proteins, ranging from conventional to deep learning approach. Further, we selected some suitable predictors from among the reviewed tools and conducted a mini comparison of their performance using a worldwide challenge dataset. Finally, we discussed the remaining major challenges in the field, and emphasized the future directions for protein function prediction with GO. 


\title{
Protein function prediction with Gene
} Ontology: from traditional to deep learning models

\author{
${ }_{4}$ Vu Thi Thuy Duong ${ }^{1}$ and Jaehee Jung ${ }^{1, *}$ \\ ${ }^{1}$ Department of Information and Communication Engineering, Myongji University, \\ Yongin-si 17058, Gyeonggi-do, Korea \\ Corresponding author: \\ Jaehee Jung* \\ Email address: jhjung@mju.ac.kr
}

10 ABSTRACT

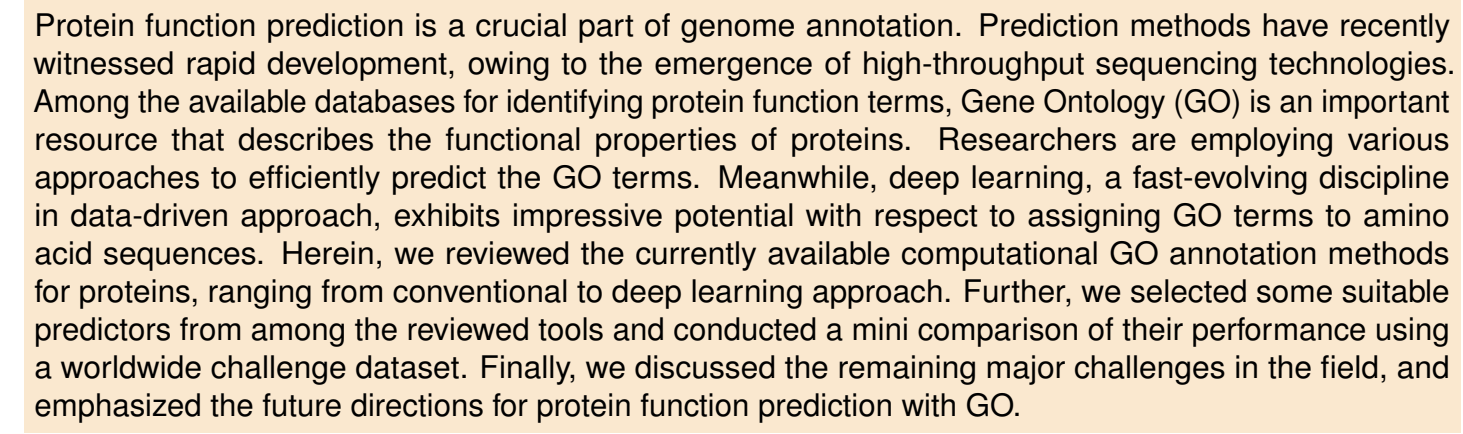

\section{INTRODUCTION}

Proteins are organic macromolecules that are fundamental determinants of the structure and function of living organisms. They play a role in numerous processes, including biochemical reactions, transmission of signals, nutrient transport, immune system boosting, etc. Therefore, understanding protein properties is essential not only from the biological and evolutionary perspectives, but also from the viewpoint of leveraging their potential in biomedical and pharmaceutical applications, and other areas (Amiri-Dashatan et al., 2018).

Generally, protein function identification is accomplished through manual or computational annotation. The former approach is the gold standard for functional annotation, because it is implemented by expert annotators and yields high quality curated results. Nonetheless, this approach is expensive and laborious, and thus, it is difficult to scale. Furthermore, because of the development of high-throughput sequencing technologies, such as next-generation sequencing (NGS), the amount of sequences to be annotated has increased dramatically. Thus, computational annotation methods have been developed as a must to automatically process the high volume of newly generated sequences, and also to improve the accuracy of the annotated data (Sleator and Walsh, 2010).

Because of the variability in the vocabulary used to define protein function, which makes the annotation process confusing to both humans and machines, various databases have been proposed to provide a standardized scheme, such as the Enzyme Commission (EC) (ENTRIES, 1995), Functional Catalogue (FunCat) (Ruepp et al., 2004), and Kyoto Encyclopedia of Genes and Genomes (KEGG) (Kanehisa et al., 2004). Currently, Gene Ontology (GO) (Ashburner et al., 2000; Consortium, 2015) is the most comprehensive resource, as it possesses all the desirable properties of a functional classification system (Pandey et al., 2006). The GO consortium created a database for a controlled vocabulary describing the functional properties of genomic products (e.g., genes, proteins, and RNA). Each ontology (vocabulary) belongs to one of three categories: molecular function (MF), biological process (BP), and cellular 
component (CC). In terms of structure, the GO follows a hierarchical organization as a directed acyclic graph (DAG), in which each term is a node and each edge connected to two nodes represents a parent-child relationship. It can be used to infer many types of information, such as "is-a" or "part-of". For instance, if term A is denoted as "is-a" of term B, it means that A is a sub-type of B. Further, "part-of" implies that the child node is necessarily part of the parent. This allows the flexible annotation of proteins with respect to the various levels of function-from general to specific terms-depending on the available evidence (Stein, 2001).

Automated function prediction (AFP) based on the GO system is a challenging problem in bioinformatics. Many studies have discussed protein functional annotation from different perspectives. Previous reviews focused on AFP (Rost et al., 2003) in terms of the data type used (Watson et al., 2005; Pandey et al., 2006; Sleator and Walsh, 2010; Shehu et al., 2016), drawbacks and corresponding solutions (Friedberg, 2006), protein interaction networks (Sharan et al., 2007), types of classified function (Rentzsch and Orengo, 2009), and GO assignment based on sequence information (Cozzetto and Jones, 2017). The two latest reviews are prepared by Bonetta and Valentino (2020) and Zhao et al. (2020). Bonetta and Valentino (2020) demonstrate protein function prediction in the machine learning workflow. Meanwhile, Zhao et al. (2020) discuss prediction of gene function prediction from the GO modeling perspective. All these researches provided independent perspectives to the issue, but we observed that there has not been any detailed review about deep learning, which is an emerging approach for protein function prediction with GO terms. Therefore, our paper suggests the possibility of predicting protein function using both conventional learning and deep learning, further indicating that better predictive performance can be expected by comparing several methods with each other.

Herein, we reviewed automated protein function prediction using GO terms (ranging from traditional solutions to the most recently developed deep learning-based tools). We presented not only an overview of the literature, but also, a performance comparison of the emerging solutions. Further, we highlighted the challenges and future prospects of the field. We hope that this review will provide bioinformaticians, computer scientists or any researchers who are interested in this topic, with the latest updates in terms of protein features used, models, and evaluation criteria, hoping to contribute to further improvements in the future.

\section{SURVEY METHODOLOGY}

Based on previous studies (Jung and Thon, 2006; Jung et al., 2010) and the aforementioned surveys, our review is divided in two main parts offering an overview of the field (from its inception to its present state). The first parts covers the conventional approach and includes solutions that do not use deep learning, while the second part describes methods that rely on deep learning to address the problem of protein functional annotation. We used Google Scholar https://scholar.google.com/ as the literature database to retrieve relevant publications, without applying any restrictions as to the publishing data, journal, or publisher.

Below, we review the conventional approach briefly, as some excellent literature reviews are already available on the subject (Shehu et al., 2016; Cozzetto and Jones, 2017). In that part, we summarize three main sub-categories of the traditional approach, and mention prominent or most recent corresponding studies. The second part of the review is our main focus. Search results using combinations of the following keywords: "deep learning", "deep neural network", "Gene Ontology", "GO terms", "protein function prediction", "functional annotation", and "gene function prediction", are selected after screening the titles, abstracts and conclusions. We also referred to references cited in the downloaded papers, to capture significant studies in the field.

\section{CONVENTIONAL APPROACH FOR PREDICTING PROTEIN GO TERMS}

In general, several techniques have been proposed to tackle the assignment of GO terms to proteins using different types of data and methods. We review the representative solutions below, focusing on three categories: similarity-based methods, probabilistic methods, and machine learning methods.

\section{Similarity-based methods}

Initially, functional annotations were assigned to uncharacterized proteins based on a simple principle: a protein sequence was searched in databases of experimentally curated proteins and if there were any 
similar proteins retrieved ("similar" in a specifically defined way), GO terms associated with the retrieved sequences were assigned to the query protein.

The very first solution of similarity-based methods was homology-based. These tools relied on local alignment search tools, such as the Basic Local Alignment Search Tool (BLAST) (Altschul et al., 1997). In these methods, the unknown sequence is searched in a database that curates well-annotated proteins. Then, a retrieved sequence with the highest alignment score-as per a predetermined threshold-is identified and its annotation is transferred to the query protein. OntoBlast (Zehetner, 2003), GOFigure (Khan et al., 2003), GOblet (Hennig et al., 2003), and GOtcha (Martin et al., 2004) are typical annotation systems adopting sequence similarity determined by BLAST search. Further details regarding these tools are presented in Table 1.

PFP (Hawkins et al., 2009) is another method that utilizes functional information associated with remote homologs by employing Position-Specific Iterated BLAST (PSI-BLAST) (Altschul et al., 1997). The tool is an improvement in terms of coverage and accuracy, as determined by analyzing a benchmark dataset, compared with GOtcha, top PSI-BLAST, and InterProScan (Zdobnov and Apweiler, 2001). Another variant solution based on sequence alignment is INGA (Piovesan et al., 2015), in which protein-protein interaction (PPI) network data is combined with domain assignment and sequence similarity from BLAST, to attain a consensus prediction of GO functions using enrichment analysis. Further, GoFDR (Gong et al., 2016) takes relevant GO terms from multiple sequence alignment (MSA) querying via BLAST or PSI-BLAST search. The probability of assigning a term to the query sequence is determined by the functionally discriminating residues (FDRs), a position-specific scoring matrix (PSSM) for the FDRs, and a score-to-probability table prepared using training sequences.

Although methods based on a local alignment search are straightforward and perform well to some extent, they also have some drawbacks, including database annotation errors or excessive function transfer, threshold relativity, and low sensitivity or specificity (Sasson et al., 2006). In addition to the alignmentbased methods, several other predictors are available that transfer function annotations based on the similarity at the level of protein structure, protein family, or phylogenomics (Shehu et al., 2016).

\section{Probabilistic methods}

A number of probabilistic models have been devised for protein function deduction (Deng et al., 2004a,b; Letovsky and Kasif, 2003; Nariai et al., 2007). Letovsky and Kasif (2003) employed a functional linkage graph (Marcotte et al., 1999) constructed based on a PPI network of the yeast Saccharomyces cerevisiae. The working assumption was that the probability of sharing functions between (nodes) proteins in close proximity on the graph is higher than that for nodes that are not in close proximity. In this method, the probabilities of GO terms assigned to a protein sequence are derived from a binomial model incorporating the Markov Random Field (MRF) algorithm. Later, Nariai et al. (2007) integrated multiple sources of evidence (PPI, gene expression data, protein motif information, mutant phenotype data, and protein localization data) using a Bayesian framework, to improve the prediction performance, compared with their model that only uses PPI.

Working on the same model organism, S. cerevisiae, as used in the methods described above, Kourmpetis et al. (2010) suggested applying a Bayesian approach to the MRF model. This suggestion was considered an improvement for the estimation of model parameters and the provision of predictions from network data. Further, Pinoli et al. (2015) compared different weighting schemes as combined with three algorithms, namely, truncated singular value decomposition (tSVD), semantically improved tSVD (SIM), and probabilistic latent semantic analysis with normalization (pLSAnorm). The first two methods are based on linear algebra, while the latter is a modified probabilistic model based on Bayesian inference. These methods were successfully used to generate novel GO annotations for three model organisms, i.e., Bos taurus, Danio rerio, and Drosophila melanogaster.

\section{Machine learning methods}

Machine learning-based tools have been developed to identify the hidden relationships between various protein features (sequence, structure, or other related evolutionary evidence) and functional labels, based on a training set (a group of fully characterized macromolecules), and use that information to generate annotations for novel proteins. Various methods that rely on machine learning have been suggested, incorporating different lines of evidence as features to train the classifiers and predict GO terms. Branch (a) of Figure 1 formulates the common workflow of those solutions, the details of which can vary between methods as described below. 
Table 1. GO annotation predictors using the traditional approach

\begin{tabular}{|c|c|c|c|}
\hline Methods & $\begin{array}{l}\text { Program } \\
\text { name }\end{array}$ & Description & Reference \\
\hline \multirow{7}{*}{ 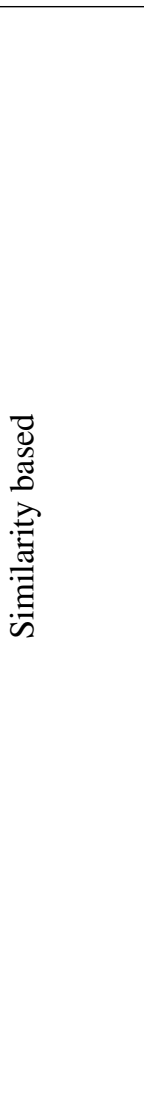 } & OntoBlast & $\begin{array}{l}\text { Web server, which is a part of the "Ontologies TO } \\
\text { GenomeMatrix" tool (Hewelt et al., 2002). It predicts } \\
\text { protein function by a weighted list of GO terms associated } \\
\text { with BLAST hit sequences in nine genome databases. }\end{array}$ & $\begin{array}{l}\text { (Zehetner, } \\
\text { 2003) }\end{array}$ \\
\hline & GOFigure & $\begin{array}{l}\text { Produces output as a clickable graph in four steps, includ- } \\
\text { ing homologous sequence search, minimum cover graph } \\
\text { construction, and assigning ontologies after scoring them. }\end{array}$ & $\begin{array}{l}\text { (Khan et al., } \\
\text { 2003) }\end{array}$ \\
\hline & GOblet & $\begin{array}{l}\text { Software package allowing the user to define the E-value } \\
\text { sensitivity and databases used for the BLAST search. All } \\
\text { ontologies identified after matching the proteins are con- } \\
\text { structed in a summary DAG structure, where the number } \\
\text { of sequences sharing a common GO term is cumulative to } \\
\text { present the significance of the term. }\end{array}$ & $\begin{array}{l}\text { (Hennig et al., } \\
\text { 2003) }\end{array}$ \\
\hline & Gotcha & $\begin{array}{l}\text { Transfers GO associations retrieved from BLAST to gene } \\
\text { products through a novel ranking scheme. The E-values } \\
\text { of the derived terms are propagated to their parent terms } \\
\text { on DAG, providing normalized confidence scores for an } \\
\text { individual GO ontology. }\end{array}$ & $\begin{array}{l}\text { (Martin et al., } \\
\text { 2004) }\end{array}$ \\
\hline & PFP & $\begin{array}{l}\text { Functional annotation system based on PSI-BLAST hits, } \\
\text { exploiting matched sequences up to an unconventionally } \\
\text { high threshold, and mining highly relevant GO terms from } \\
\text { UniProt to score functions for unknown proteins. }\end{array}$ & $\begin{array}{l}\text { (Hawkins } \\
\text { et al., 2009) }\end{array}$ \\
\hline & INGA & $\begin{array}{l}\text { Functional annotation server derives GO predictions from } \\
\text { combining many data sources. }\end{array}$ & $\begin{array}{l}\text { (Piovesan } \\
\text { et al., 2015) }\end{array}$ \\
\hline & GoFDR & $\begin{array}{l}\text { Multiple alignment-based method utilizing FDRs and } \\
\text { PSSM to rank GO terms for amino acid sequences. }\end{array}$ & $\begin{array}{l}\text { (Gong et al., } \\
\text { 2016) }\end{array}$ \\
\hline \multirow[t]{2}{*}{ 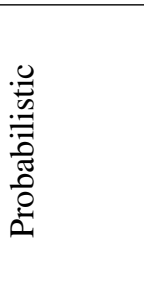 } & - & $\begin{array}{l}\text { Probabilistic framework based on PPI, predicting GO as- } \\
\text { signments for } 10 \% \text { unannotated proteins in yeast. } \\
\text { Leverages heterogeneous data to increase the probabilis- } \\
\text { tic based model for the functional prediction of Saccha- } \\
\text { romyces cerevisiae. }\end{array}$ & $\begin{array}{l}\text { (Letovsky and } \\
\text { Kasif, 2003) } \\
\text { (Nariai et al., } \\
\text { 2007) }\end{array}$ \\
\hline & BMRF & $\begin{array}{l}\text { Variant of the MRF-based approach on PPI that infers } \\
\text { novel predictions for unannotated proteins. }\end{array}$ & $\begin{array}{l}\text { (Kourmpetis } \\
\text { et al., 2010) }\end{array}$ \\
\hline \multirow{6}{*}{ 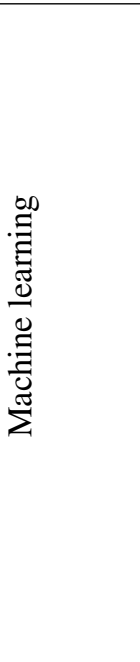 } & GOPET & $\begin{array}{l}\text { GO term prediction and evaluation tool, supplying molec- } \\
\text { ular function and biological process GO terms for any } \\
\text { organism. }\end{array}$ & $\begin{array}{l}\text { (Vinayagam } \\
\text { et al., 2006) }\end{array}$ \\
\hline & PoGO & $\begin{array}{l}\text { Ensemble model with InterPro sequence similarity, bio- } \\
\text { chemical property, and protein tertiary structure to predict } \\
\text { the GO of fungal proteins. }\end{array}$ & $\begin{array}{l}\text { (Jung et al., } \\
2010 \text { ) }\end{array}$ \\
\hline & FFPred3 & $\begin{array}{l}\text { SVM-based tool, providing the homology-independent } \\
\text { assignment of GO terms for eukaryotic proteins. }\end{array}$ & \\
\hline & PANNZER2 & $\begin{array}{l}\text { Weighted } k \text {-nearest neighbor classifier, providing func- } \\
\text { tional annotation for uncharacterized proteins with GO } \\
\text { terms and free text descriptions. }\end{array}$ & $\begin{array}{l}\text { (Törönen } \\
\text { et al., 2018) }\end{array}$ \\
\hline & DeepText2GO & $\begin{array}{l}\text { Combination of a text-based method and a sequence-based } \\
\text { method to improve large-scale protein function prediction. }\end{array}$ & $\begin{array}{l}\text { (You et al., } \\
\text { 2018a) }\end{array}$ \\
\hline & NetGo & $\begin{array}{l}\text { Effective LTR-based web server, combining both sequence } \\
\text { and massive network information of proteins to annotate } \\
\text { gene products. }\end{array}$ & $\begin{array}{l}\text { (You et al., } \\
2019 \text { ) }\end{array}$ \\
\hline
\end{tabular}




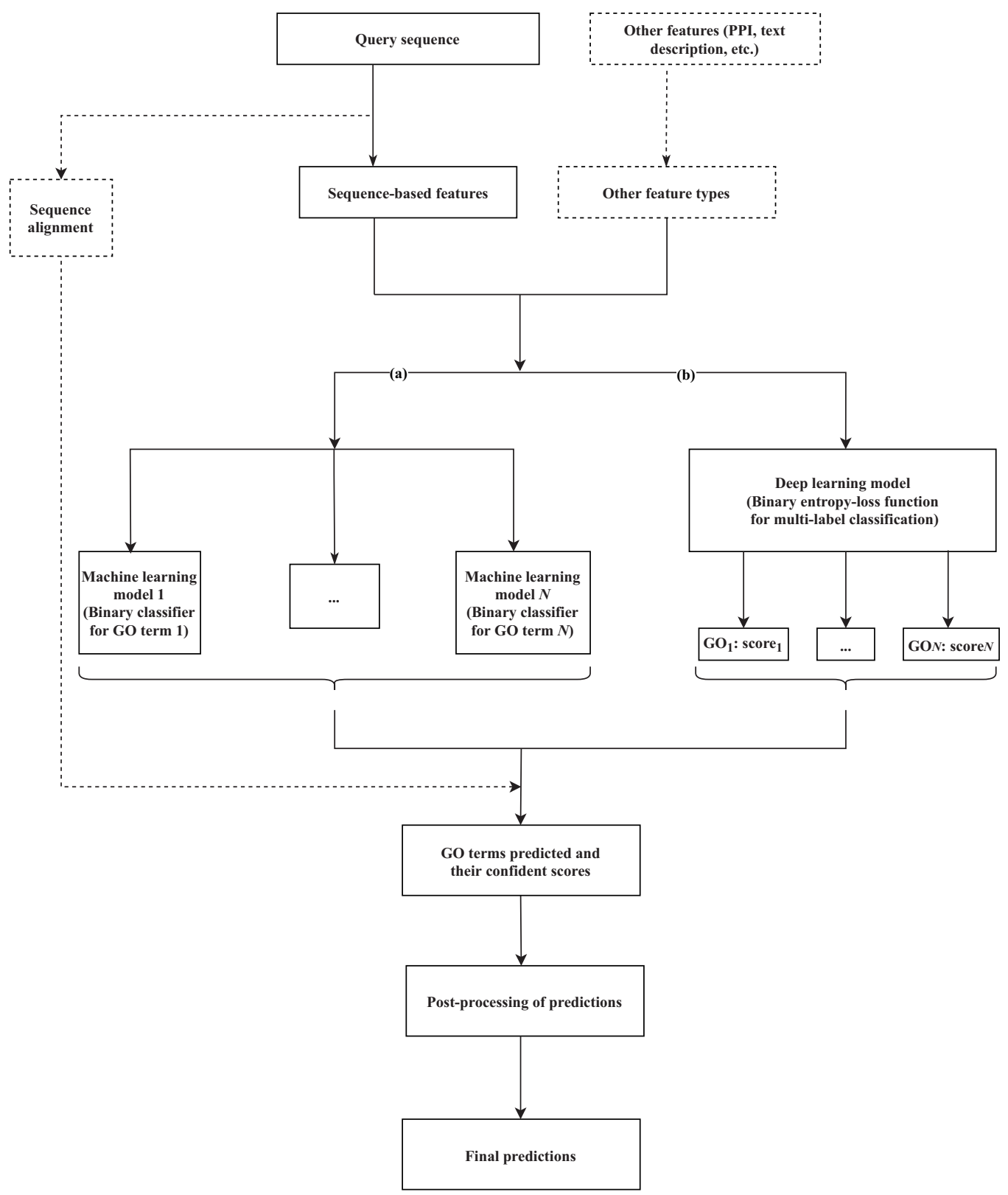

Figure 1. A common workflow of (a) machine learning-based and (b) deep learning-based solutions for predicting the GO terms of proteins. The components presented in the dashed box are optional, depending on each method. $N$ is the number of GO terms that are selected for the training dataset to build the model. 
Utilizing multiple support vector machine (SVM) classifiers is a predominant selection in several studies. For instance, GOPET (Vinayagam et al., 2006) feeds several features related to GO terms (sequence similarity measures based on BLAST search, frequency, quality of homolog annotation, and annotation level within the GO hierarchy) to 99 SVM classifiers. A specific term will be predicted to be a "correct" or "incorrect" label for an unknown sequence, with voting confidence scores calculated. As another example, FFPred (Lobley et al., 2007, 2008) was initially established for the unannotated human proteome, but demonstrated a generalization to other proteomes. The latest version of FFPred is FFPred3 (Cozzetto et al., 2016), which is still based on SVM, is expanded to investigate the correlations between feature characteristics retrieved from sequences and structures within the three sub-ontologies (MF, BP, and CC). Another tool, Prediction of Gene Ontology terms (PoGO) (Jung et al., 2010), has been developed from Automatic Annotation of Protein Functional Class (AAPFC) (Jung and Thon, 2006). Instead of only using InterPro terms as features, PoGO integrates three more sources (sequence similarity, biochemical properties, and protein tertiary structure). Subsequently, SVM and a linear classifier (Freund and Schapire, 1997) are used as base-level classifiers before the meta-learning step.

Using the $k$-nearest neighbor $(k$-NN) algorithm, PANNZER2 (Törönen et al., 2018) provides a fast functional annotation systems based on sequence homology and other annotation predictors. Meanwhile, MS- $k$ NN (Lan et al., 2013) aggregates heterogeneous data to propose a competitive model for protein function prediction. DeepText2GO (You et al., 2018a) is a consensus approach, integrating deep semantic text representation from MEDLINE citations (ncb, 2018) as text information, and sequence information obtained via BLAST and InterProScan. These features are fed to the $k$-NN and logistic regression models to produce the functions of no-knowledge proteins on a large scale. NetGO (You et al., 2019) is an extension of GoLabeler (You et al., 2018b), which employs the learning-to-rank (LTR) model to integrate sequence-based evidence. NetGO improves the performance of a large scale AFP by accessing the enormous protein-protein network of over 2000 species in the STRING database (Szklarczyk et al., 2016).

Machine learning approaches are highly advantageous and considered the future direction for AFP. Some novel proteins either lack identifiable homologous sequences or their detectable homologues have not been assigned any GO labels. Consequently, assigning protein function from scratch using machine learning, i.e., directly inferring the annotation from the amino acid sequence, without access to any additional references or databases, is an ongoing task. The sophisticated algorithms are being developed, as presented in the next section.

\section{GO TERM ANNOTATION OF PROTEINS USING DEEP LEARNING APPROACH}

The excellent potential of deep learning — the latest discipline of machine learning — has been demonstrated in several application fields, including bioinformatics (Min et al., 2017; Li et al., 2019). The main characteristic that distinguishes this approach from other methods is the learning process. Deep learning models are inspired by neural networks in the human brain. They automatically extract high-level features from raw data and provide predictions in an end-to-end manner. In contrast to that in conventional machine learning models, accurate classification is achieved via handcrafted features.

Currently, the amount of the generated genomic data, and the numbers of sophisticated algorithms and computational resources are rapidly growing. These resources support deep learning to tackle the functional annotation problem. Below, we review proposed methods based on two criteria, i.e., the model and the input data used. A summary table of the reviewed methods is presented in Table 2, and a common workflow for deep learning approach is illustrated as branch (b) of Figure 1.

\section{Model-based approach Supervised learning}

Supervised learning is an essential approach for in silico protein function prediction. Supervised models learn to annotate function guided by a training dataset, which is a group of characterized proteins with reliable, experimentally confirmed annotations. After the learning stage, a model that captures the relationship between feature and function is produced, and used to predict the GO terms for novel amino acid sequences.

Deep neural network (DNN) is a feed-forward architecture. It generally comprises an input layer, multiple hidden layers, and an output layer. Input data are processed in a unidirectional manner along the layers, from the first to the final stage. With respect to GO-based functional annotation, DNNs have 
Table 2. Deep learning-based methods for assigning GO terms to proteins

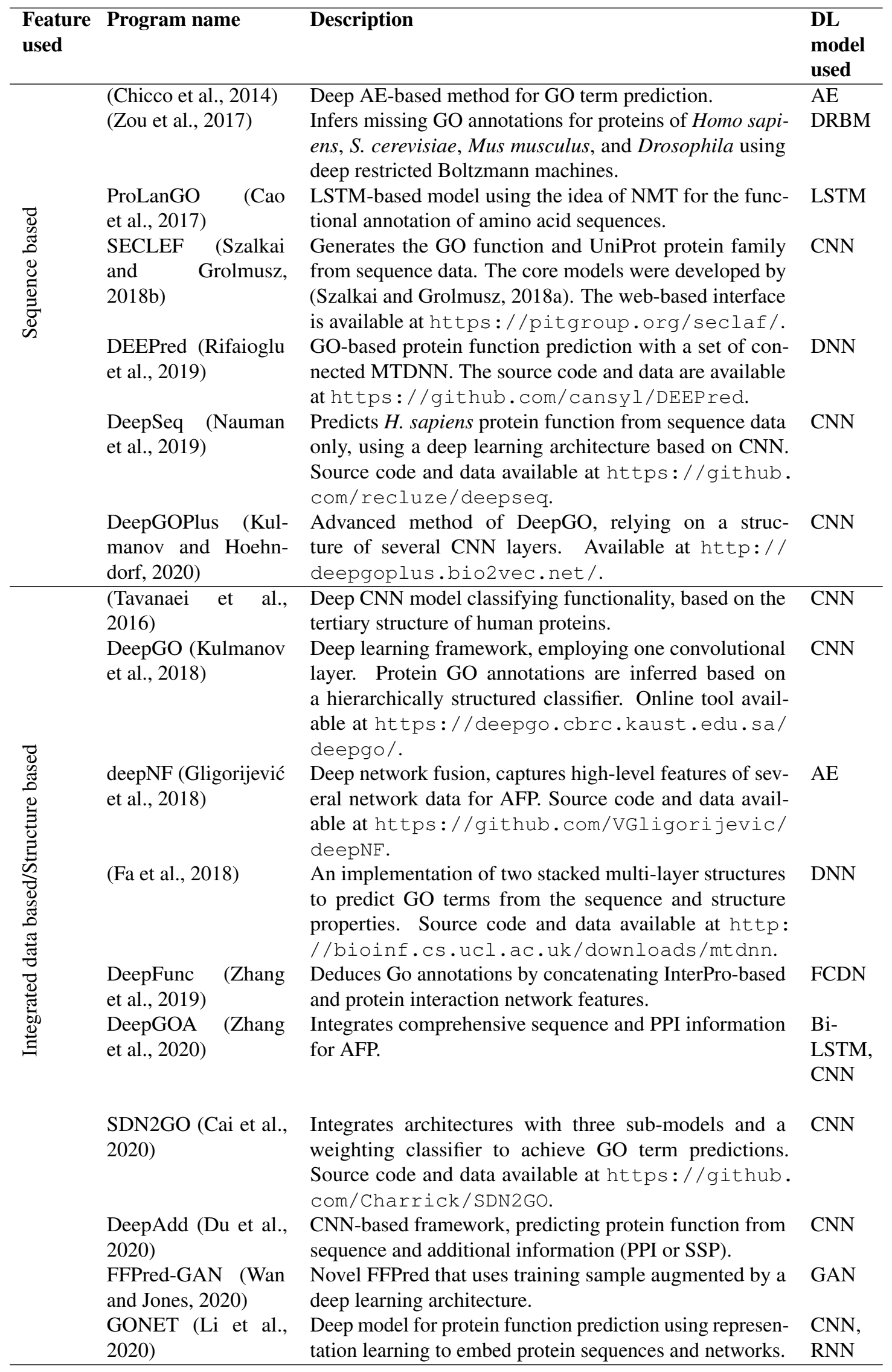


been employed as multi-task DNNs (MTDNNs) (Fa et al., 2018) or a series of MTDNNs (Rifaioglu et al., 2019).

Convolutional neural network (CNN) was initially developed to handle two-dimensional images for handwritten digit recognition (LeCun et al., 1990). However, it has since become an effective architecture not only for multi-dimensional data, but also for one-dimensional input, such as sentences or genomic sequences. A CNN model starts with convolutional layers, whose units are feature maps. Each unit is obtained by computing convolution operations between local patches of a unit in the preceding layer and a filter (set of weights) (LeCun et al., 2015). Non-linearity pooling layers are stacked to convolutional layers, enabling the integration of different-scale features in this architecture. CNNs can be applied in AFP either alone (Kulmanov et al., 2018; Cai et al., 2020; Du et al., 2020) or in combination with other architectures (Zhang et al., 2020; Spalević et al., 2020).

Recurrent neural network (RNN) is a deep learning architecture developed especially for sequential data. RNNs have a DNN backbone, and the units of the hidden layer are connected. Therefore, a hidden unit receives information from the input layer at the current time step, and also from the hidden units at the preceding stage (LeCun et al., 2015). Long short-term memory (LSTM) (Hochreiter and Schmidhuber, 1997) is a variant of the RNN model, developed to capture long-term dependencies between input sequences. Bi-directional LSTM (Bi-LSTM) is an LSTM model that processes data in two directions, forwards and backwards. These neural networks are employed to process protein sequences as a natural language (Cao et al., 2017) or are combined with the CNN model (Zhang et al., 2020) to provide GO annotations.

Another type of neural network, fully connected deep network (FCDN), is a series of fully connected layers. This model was utilized for converting input vectors from InterPro and predicting GO terms (Zhang et al., 2019).

\section{Unsupervised learning}

Unlike supervised learning, unsupervised learning independently mines hidden patterns in input distribution. It is crucial for exploring unlabeled data and provides important information for supervised architectures. Typically, unsupervised learning models are employed for clustering, reducing dimensions, and transforming data.

Autoencoder (AE) (Baldi, 2012) is an unsupervised learning model developed for assigning GO terms to amino acid sequences. AE is a neural network for data transformation. After encoding and decoding, the target output size is the same as that of the input. Using a trained AE model, GO predictions for proteins can be deduced directly from the generated matrix (Chicco et al., 2014). Alternatively, low-dimensional feature representations in the hidden layer are extracted and fed into an SVM classifier for final classification (Gligorijević et al., 2018) or a CNN model (Peng et al., 2020) for final classification.

Restricted Boltzmann machine (RBM) (Salakhutdinov and Hinton, 2009) has one hidden layer for representing latent features and an input layer encoding the observed data. For GO annotation, units of the input layer are the available GO terms (Zou et al., 2017). Zou et al. (2017) introduced a deep RBM (DRBM), where multi-layer RBMs are trained and unfolded, resulting in protein function prediction.

Finally, generative adversarial network (GAN) (Goodfellow et al., 2014) comprises two networks, i.e., a generative model that produces synthetic realistic data and a discriminative model that evaluates whether the data are real or not. In AFP, the GAN model improves the prediction by creating synthetic features for supervised classifiers (Wan and Jones, 2020), or by exploiting ontology correlations (Seyyedsalehi et al., 2021).

\section{Data-based approach}

In terms of the input feature, deep learning-based methods primarily use two approaches to assign GO terms to unknown gene products. One is a sequence-only approach, which is useful for predicting the functions of novel proteins in the absence of homologous information or other references. The second one is structure-based or otherwise exploits big data from several available resources.

\section{Sequence-based models}

One of the earliest deep learning-based methods for $\mathrm{GO}$ annotation predictions is the one proposed by Chicco et al. (2014). The authors compared two annotation solutions, i.e., tSVD, presented in (Pinoli et al., 2013), and the AE neural network. The output form of the two methods was similar; however, the latter performed better on six different datasets. Later, instead of using the AE architecture, (Zou 
et al., 2017) proposed DRBM for annotating the gene products of four model species, Homo sapiens, S.cerevisiae, Musmusculus, and Drosophila.

ProLanGo (Cao et al., 2017) is the first tool that applies Neural Machine Translation (NMT) developed by Google in AFP. The authors converted amino acid sequences and GO terms into "ProLan" and "GOLan" languages, respectively. The GO annotation of a new protein was then generated after being translated using a model with three RNN layers. In another study, artificial neural networks proposed by Szalkai and Grolmusz (2018a) utilized six CNN layers of increasing depth. Based on that work, SECLEF (Szalkai and Grolmusz, 2018b) has been designed to train and test the GO function and UniProt protein family using biological sequences as input. The user can implement their self-configured models in a downloadable program or run two pre-trained models (Szalkai and Grolmusz, 2018a) on a web server. DeepSeq (Nauman et al., 2019) is another method for assigning GO terms to amino acid sequences, based on convolutional layers. However, the authors only predicted the five most frequent MF ontologies for $H$. sapiens proteins.

Using the DNN architecture, DEEPred (Rifaioglu et al., 2019) implements a stack of multi-task feed-forward DNNs. Each individual network is built for a specific GO term level on DAG, which allows the hierarchical post-processing of predictions. Protein sequences are experimentally represented by three types of descriptors (subsequence profile map, pseudo-amino acid composition, and conjoint triad feature), with subsequence profile map performing best in the analysis.

DeepGOPlus (Kulmanov and Hoehndorf, 2020) has been developed to overcome the existing limitations of DeepGO (Kulmanov et al., 2018), such as sequence length, unavailable PPI features, and the number of GO labels. Functional annotation is predicted by a multi-layer CNN structure in combination with sequence similarity. Lately, TALE (Cao and Shen, 2021) has been developed to generate GO predictions by integrating sequence patterns based on transformer encoder and the joint similarity of sequence-term. Similar to DeepGOPlus, TALE is also combined with sequence similarity as TALE+ model to enhance its performance. Meanwhile, PFP-WGAN (Seyyedsalehi et al., 2021) is one of two latest ideas that use GAN to infer the functionalities of proteins. While the generator network processes raw sequences, the discriminator takes two inputs; one consists of annotated proteins from the SwissProt database and the other of raw sequences with respective annotations synthesized from the generator.

\section{Integrated data-based/Structure based-models}

The functional assignment of amino acid sequences from 3D structures was proposed by (Tavanaei et al., 2016). The main analysis involved a CNN model that was trained and tested on five datasets of human proteins, each of which had been assigned two GO terms. However, the prediction has not been propagated on the DAG tree for inherited GO terms.

DeepGO (Kulmanov et al., 2018) is a functional annotation server based on CNN architecture that had been devised to learn features from amino acid sequences and the PPI network. GO labels are assigned to input proteins through a hierarchical classification structured as a DAG tree. DeepAdd (Du et al., 2020) was inspired by DeepGO server and provides a solution for AFP, utilizing a CNN framework to learn vector representations from sequences and additional information. However, sequences are processed into $k$-mer embedding by Word2Vec, instead of the tri-gram embedding suggested in DeepGO. Further, in DeepAdd, the protein sequence profile (SSP) is added if the PPI network information is not available. Based on a similar concept of combining primary protein structure and PPI, GONET (Li et al., 2020), a novel model, was built by employing CNN, RNN, and Attention layer for human and mouse sequences.

Working on a dataset identical to the one collated by FFPred3, Fa et al. (2018) tested an MTDNN that treated protein function prediction as a multi-label classification problem. Here, the solution consisted of layers shared by all tasks (GO labels), which are stacked in parallel with task-specific layers. DeepFunc (Zhang et al., 2019) is a novel predictor that surpasses DeepGO, FFPred3, and BLAST. First, protein domain, family, and motif information is queried from InterPro and encoded before passing through fully connected layers. Next, topological features of PPI are obtained by the Deepwalk algorithm. Finally, the method concatenates two types of features (sequence- and network-based input) to fit an FCDN. The architecture of DeepGOA (Zhang et al., 2020) is more sophisticated than that of DeepFunc. In addition to information generated by InterPro and PPI, global and local semantic features of amino acid sequences are extracted by Bi-LSTM and a convolutional layer, respectively. Using the same types of features, SDN2GO (Cai et al., 2020) utilizes three sub-models for each information source, with all outputs integrated in the final weighted model.

In another study, deepNF (Gligorijević et al., 2018) was constructed by multimodal deep AE to capture hidden information in proteins from different types of interaction networks. In that suggestion, 
Table 3. Statistics of final benchmark CAFA3

\begin{tabular}{l|c|c|c|c}
\hline \multirow{2}{*}{ Annotation type } & \multirow{2}{*}{ Number of sequences } & \multicolumn{3}{|c}{ Number of terms (GO leaf only) } \\
\cline { 3 - 5 } & & MF & CC & BP \\
\hline NK (no-knowledge) & 1177 & 377 & 236 & 1087 \\
\hline LK (limited-knowledge) & 2151 & 368 & 203 & 878 \\
\hline All (NK + LK) & 3328 & 631 & 327 & 1673 \\
\hline
\end{tabular}

feature representations are extracted to train the final SVM classifier to produce GO terms for human proteins. DeepMNE-CNN (Peng et al., 2020) has a superior performance than deepNF in the human data by utilizing CNN layers instead of SVM for the classification model. On the other hand, FFPred-GAN (Wan and Jones, 2020) utilizes GAN for feature enrichment to feed conventional machine learning models, especially SVM. Real features are biophysical information extracted from raw amino acid sequences by FFPred, and the generator takes latent variables to augment the synthetic samples.

\section{COMPARISON}

In addition to proposing a new approach, accuracy assessment is important for demonstrating the improvements of a novel methodology. This necessitates the establishment of a common framework for the evaluation and comparison of the proposed solutions. Accordingly, the critical assessment of functional annotation (CAFA) (Radivojac et al., 2013; Jiang et al., 2016; Zhou et al., 2019) is a community-based experiment that provides a large-scale evaluation of computational protein function prediction methods in a time-delayed manner. Four main competitions (CAFA1-CAFA4) have been held every 3 years since 2010. CAFA- $\pi$ is an extension of CAFA3, and allows the competitors to improve their computational solutions focusing on the prediction of genes associated with specific GO terms. Generally, each challenge comprises three phases, i.e., predictions for a set of target proteins, experimental verification for a subset of target proteins to obtain a benchmark dataset, and performance analysis of all the competitors based on predefined metrics and the benchmark data. Official reports have just been released for the first three challenges and CAFA- $\pi$ (Radivojac et al., 2013; Jiang et al., 2016; Zhou et al., 2019).

In the remainder of this section, we present a practical comparison of emerging analysis methods and discuss the retrieved results.

\section{Set up \\ Data}

We used the finalized benchmark set provided in the CAFA3 report (Zhou et al., 2019). The statistics are presented in Table 3. There are two sequence types in the benchmark dataset, NK (no-knowledge) and LK (limited-knowledge), which have been coined in CAFA2 (Jiang et al., 2016). Based on the timeline of the challenge, $t_{0}$ is the deadline for the prediction submission of the target sequences. Then, experimental annotations for a subset of target sequences are accumulated until $t_{1}$, to complete a benchmark dataset for the performance evaluation. The NK proteins are those that had not had any experimental annotations in three GO domains at $t_{0}$, but had at least one GO term with experimental evidence code between $t_{0}$ and $t_{1}$. The LK sequences are those that had been experimentally annotated in one or two GO domains at $t_{0}$, and gained at least one experimental annotation in the other GO domains between $t_{0}$ and $t_{1}$.

\section{Methods}

The servers of several tools have been updated recently, but we need to control their training data or databases used, in which do not include sequences in the CAFA3 benchmark. Therefore, we chose INGA, which was one of the top models in CAFA3, as a representative conventional tool. DeepGOPlus is a recently developed representative annotation system that is based on deep learning. In addition to these two tools, other studies that performed their analysis on the CAFA3 dataset are also summarized in this part.

For DeepGOPlus, we re-trained the model with the CAFA3 training sequences and generated predictions for the CAFA3 benchmark set based on their source code provided. For INGA, we obtained predictions for the CAFA3 benchmark based on the predictions of CAFA3 targets, which were provided on their website. 

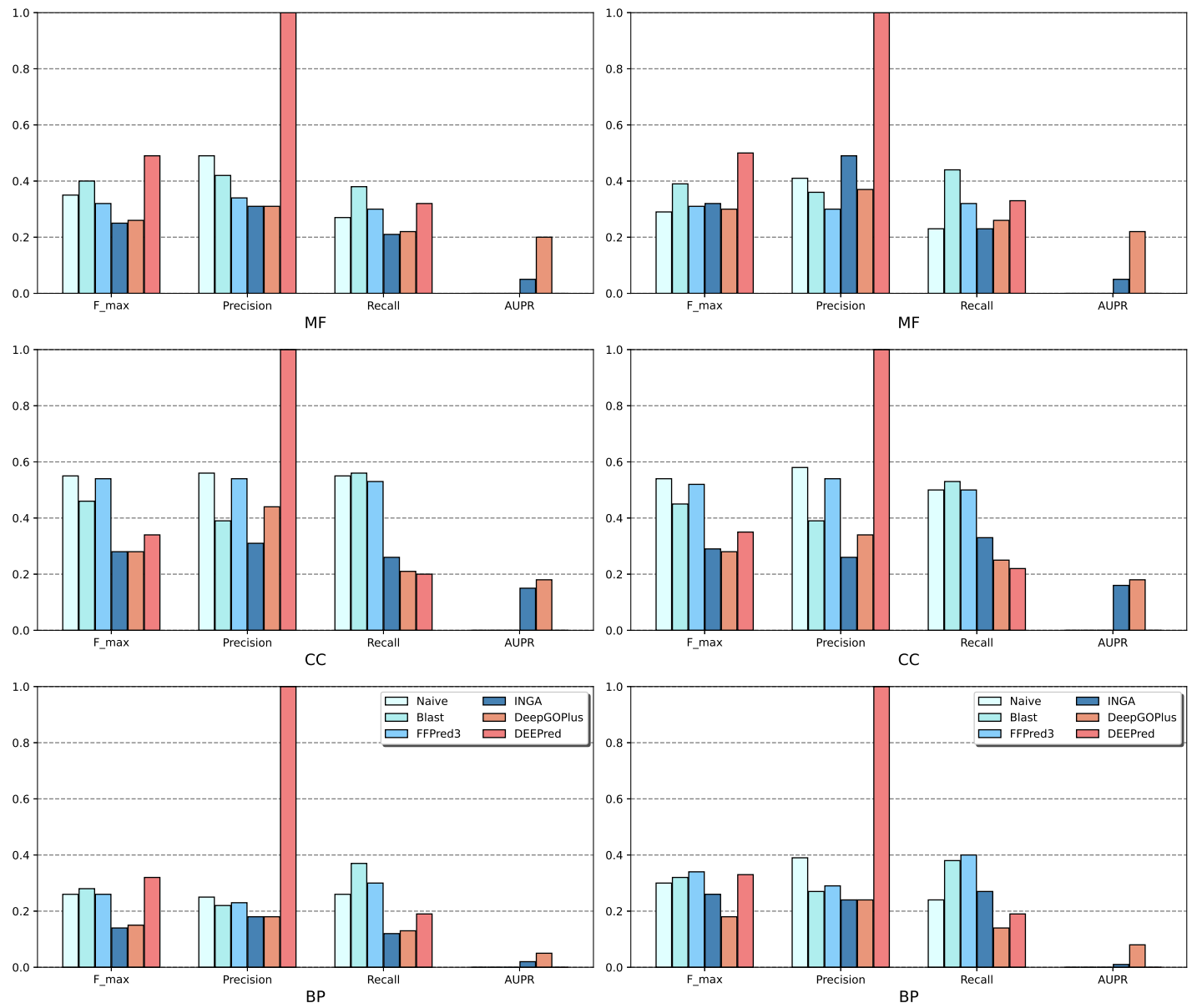

A

B

Figure 2. Performance of the selected methods with the CAFA 3 benchmark dataset in full mode: (A) NK sequences and (B) All sequences (NK + LK). Scores in the full mode were computed on all protein sequences in the benchmark set. Scores of Naive, BLAST, FFPred3, DEEPred have been reported directly from (Rifaioglu et al., 2019).

Model performances were evaluated based on protein-centric metrics ( $F_{\max }$, precision, and recall) provided by the CAFA3 assessment tool. The area under the precision-recall curve (AUPR), which is a measurement of highly imbalanced data, was also considered. Results regarding the performance of other methods, including Naive, BLAST, FFPred3 and DEEPred, were derived from DEEPred paper (Rifaioglu et al., 2019).

In terms of evaluation, two modes were designed. In the partial mode, the scores of each method were computed on the sequences that have at least one GO term predicted at any threshold. Conversely, scores in the full mode were computed on all protein sequences in the benchmark set.

\section{Results}

The results of the analysis are shown in terms of GO domains and types of sequences (NK vs. All) in full mode (Figure 2) and partial mode (Figure 3). The scores of Naive, BLAST, FFPred3 and DEEPred are only available for the full mode. Models using the traditional approach and deep learning approach are depicted in blue and orange tones, respectively.

As is shown in Figure 2, deep learning-based models (DEEPred and DeepGOPlus) showed a competitive performance compared to conventional models in terms of MF and BP prediction; in particular, DEEPred acquired the highest precision in the three GO categories. Such high precision obtained by DEEPred, which was explained in their paper (Rifaioglu et al., 2019), probably resulted from the imbalanced dataset and post-processing step. For example, the negative protein set for each GO term is 

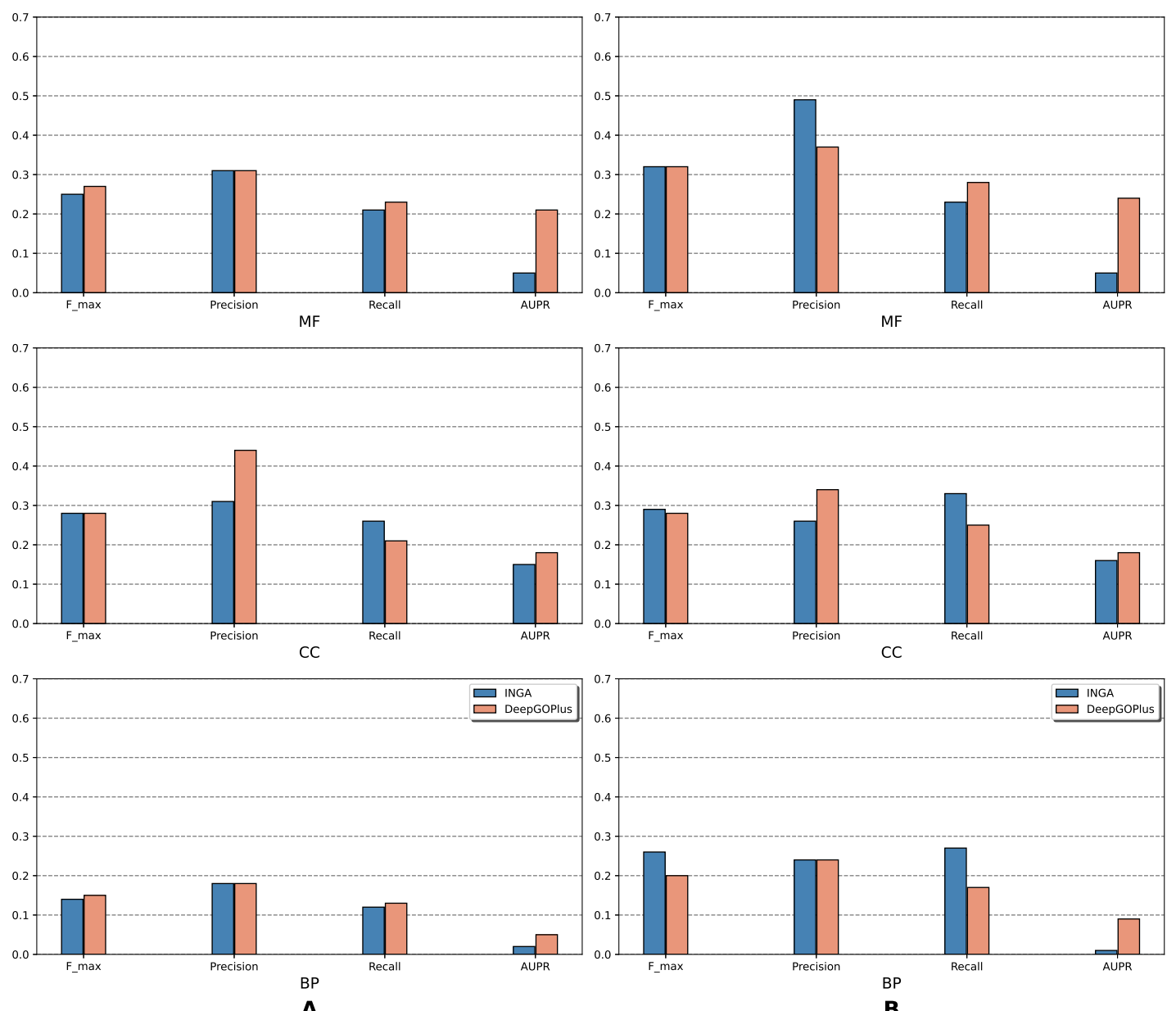

A

B

Figure 3. Performance of the selected methods with the CAFA3 benchmark dataset in partial mode: (A) NK sequences and (B) All sequences (NK + LK). In the partial mode, scores of each method were computed on the sequences that have at least one GO term predicted at any threshold. 
four times as large as the positive one, this leads to low false positive and high false negative predictions. Additionally, DEEPred filtered some false positives at the post-processing step, thus increasing their precision. For the CC class, two baselines (Naive and BLAST) were at the top for $F_{\max }$ and recall, followed by FFPred3. The overall outcome is presented similarly in Figure 3, in which DeepGOPlus performed better than INGA in most of categories.

The aforementioned results and the CAFA3 report indicate that conventional models, such as INGA and GOLabeler (referred to as "ZhuLab" in CAFA3) perform as well as other high-performing models. ZhuLab was the best performer in terms of $F_{\max }$ and NK-full evaluation, with the scores of $0.40,0.61$, and 0.62 for BP, CC, and MF, respectively. However, these conventional models integrate several types of information, which is not always available for all sequences. Meanwhile, the deep learning approach had demonstrable advantages in this area and yielded highly competitive predictions. The research direction is currently shifting toward this new approach, with even more efficient solutions being developed.

\section{CONCLUSION}

The computational GO annotation of proteins has been an actively pursued and challenging task in bioinformatics since around the 2000s; this is a response to the need to bridge the gap between the known and unknown, newly discovered amino acid sequences. On the one hand, understanding protein function is essential for deciphering biological evolution and for countless applications, such as drug design and disease treatment. The GO database has facilitated a comprehensive vocabulary for functional annotation because it presents structured GO functions in three domains (MF, BP, and CC), thereby effectively supporting in silico protein function assignment. Herein, we presented the current state of the field and compared GO-based AFP solutions, classed as conventional and deep learning approaches. As it is difficult to introduce all the available methodologies and comprehensively compare them, we tested the prominent predictors in this study. This was complemented by the analysis of related publications, with the assessment outcomes computed by CAFA, a worldwide venue for comparing computational protein function predictors.

We observed that one of the primary difficulties in the field is the utilization of input features to achieve effective performance. While heterogeneous input data are useful for GO prediction, only sequence information is available for the majority of unannotated proteins. With respect to the specific approaches, machine learning models may be limited by the heterogeneity of genomic data when mining different sources of information, while parameter and hyper-parameter tuning is the challenging step of the deep learning approach. In terms of output, the GO database has been updating because GO annotations are still imbalanced and not complete for all species. Its sophisticated structure is ideal for describing the functional roles of proteins, but also makes the prediction task a complex multi-label problem.

Nonetheless, despite the existing challenges, the quest for successful annotation will continue in many directions, driven by the community effort. Together with the constant expansion of -omics databases, data-driven methods have shown excellent applications in various areas, with a promising trend in functional annotation. Depending on the available sources, they can be based on integrated data or sequence information only, considering a hybrid approach. Some approaches have been suggested and developed to cope with imbalanced data; these include working on the subgroups in which the classes are more balanced, data augmentation using GAN, and considering evaluation metrics specified for imbalanced data, such as AUPR. The multi-label problem can be addressed via the advancement of computational resources and well-defined solutions, for example, stacking many individual solutions together. Finally, successful solutions for GO term prediction can be expanded to include other functional resources (EC, pathways, etc.), to comprehend the biological role and life science potential of proteins.

\section{ADDITIONAL INFORMATION AND DECLARATIONS}

\section{Competing Interests}

The authors declare there are no competing interests.

\section{Author Contributions}

$\mathrm{Vu}$ Thi Thuy Duong analyzed the data, prepared figures and/or tables, and approved the final draft. Jaehee Jung authored or reviewed drafts of the paper, and approved the final draft. 


\section{Data Availability}

The following information was supplied regarding data availability: https://github.com/duongvtt $96 /$

Comparison-GO-annotation-systems

\section{Funding}

This research was supported by the National Research Foundation of Korea (NRF) funded by the Ministry of Science, ICT and Future Planning (NRF-2019R1A2C1084308).

\section{REFERENCES}

(2018). Database resources of the national center for biotechnology information. Nucleic acids research, 46(D1):D8-D13.

Altschul, S. F., Madden, T. L., Schäffer, A. A., Zhang, J., Zhang, Z., Miller, W., and Lipman, D. J. (1997). Gapped blast and psi-blast: a new generation of protein database search programs. Nucleic acids research, 25(17):3389-3402.

Amiri-Dashatan, N., Koushki, M., Abbaszadeh, H.-A., Rostami-Nejad, M., and Rezaei-Tavirani, M. (2018). Proteomics applications in health: biomarker and drug discovery and food industry. Iranian journal of pharmaceutical research: IJPR, 17(4):1523.

Ashburner, M., Ball, C. A., Blake, J. A., Botstein, D., Butler, H., Cherry, J. M., Davis, A. P., Dolinski, K., Dwight, S. S., Eppig, J. T., et al. (2000). Gene ontology: tool for the unification of biology. Nature genetics, 25(1):25-29.

Baldi, P. (2012). Autoencoders, unsupervised learning, and deep architectures. In Proceedings of ICML workshop on unsupervised and transfer learning, pages 37-49. JMLR Workshop and Conference Proceedings.

Bonetta, R. and Valentino, G. (2020). Machine learning techniques for protein function prediction. Proteins: Structure, Function, and Bioinformatics, 88(3):397-413.

Cai, Y., Wang, J., and Deng, L. (2020). Sdn2go: An integrated deep learning model for protein function prediction. Frontiers in bioengineering and biotechnology, 8:391.

Cao, R., Freitas, C., Chan, L., Sun, M., Jiang, H., and Chen, Z. (2017). Prolango: protein function prediction using neural machine translation based on a recurrent neural network. Molecules, 22(10):1732.

Cao, Y. and Shen, Y. (2021). TALE: Transformer-based protein function Annotation with joint sequence-Label Embedding. Bioinformatics. btab198.

Chicco, D., Sadowski, P., and Baldi, P. (2014). Deep autoencoder neural networks for gene ontology annotation predictions. In Proceedings of the 5th ACM conference on bioinformatics, computational biology, and health informatics, pages 533-540.

Consortium, G. O. (2015). Gene ontology consortium: going forward. Nucleic acids research, 43(D1):D1049-D1056.

Cozzetto, D. and Jones, D. T. (2017). Computational methods for annotation transfers from sequence. In The Gene Ontology Handbook, pages 55-67. Humana Press, New York, NY.

Cozzetto, D., Minneci, F., Currant, H., and Jones, D. T. (2016). Ffpred 3: feature-based function prediction for all gene ontology domains. Scientific reports, 6(1):1-11.

Deng, M., Chen, T., and Sun, F. (2004a). An integrated probabilistic model for functional prediction of proteins. Journal of Computational Biology, 11(2-3):463-475.

Deng, M., Tu, Z., Sun, F., and Chen, T. (2004b). Mapping gene ontology to proteins based on proteinprotein interaction data. Bioinformatics, 20(6):895-902.

Du, Z., He, Y., Li, J., and Uversky, V. N. (2020). Deepadd: Protein function prediction from k-mer embedding and additional features. Computational Biology and Chemistry, 89:107379.

ENTRIES, N. (1995). Nomenclature committee of the international union of biochemistry and molecular biology (nc-iubmb). Eur. J. Biochem, 233(132):139.

Fa, R., Cozzetto, D., Wan, C., and Jones, D. T. (2018). Predicting human protein function with multi-task deep neural networks. PloS one, 13(6):e0198216.

Freund, Y. and Schapire, R. E. (1997). A decision-theoretic generalization of on-line learning and an application to boosting. Journal of computer and system sciences, 55(1):119-139.

Friedberg, I. (2006). Automated protein function prediction-the genomic challenge. Briefings in bioinformatics, 7(3):225-242. 
Gligorijević, V., Barot, M., and Bonneau, R. (2018). deepnf: deep network fusion for protein function prediction. Bioinformatics, 34(22):3873-3881.

Gong, Q., Ning, W., and Tian, W. (2016). Gofdr: a sequence alignment based method for predicting protein functions. Methods, 93:3-14.

Goodfellow, I. J., Pouget-Abadie, J., Mirza, M., Xu, B., Warde-Farley, D., Ozair, S., Courville, A., and Bengio, Y. (2014). Generative adversarial networks. arXiv preprint arXiv:1406.2661.

Hawkins, T., Chitale, M., Luban, S., and Kihara, D. (2009). Pfp: Automated prediction of gene ontology functional annotations with confidence scores using protein sequence data. Proteins: Structure, Function, and Bioinformatics, 74(3):566-582.

Hennig, S., Groth, D., and Lehrach, H. (2003). Automated gene ontology annotation for anonymous sequence data. Nucleic Acids Research, 31(13):3712-3715.

Hewelt, A., Ben Kahla, A., Hennig, S., Nagel, A., Himmelbauer, H., Zehetner, G., Haas, S., Vingron, M., Yaspo, M., and Lehrach, H. (2002). The genomematrix information retrieval system. In Poster Abstracts of HGM2002 (Human Genome Meeting).

Hochreiter, S. and Schmidhuber, J. (1997). Long short-term memory. Neural computation, 9(8):17351780.

Jiang, Y., Oron, T. R., Clark, W. T., Bankapur, A. R., D’Andrea, D., Lepore, R., Funk, C. S., Kahanda, I., Verspoor, K. M., Ben-Hur, A., et al. (2016). An expanded evaluation of protein function prediction methods shows an improvement in accuracy. Genome biology, 17(1):1-19.

Jung, J. and Thon, M. R. (2006). Automatic annotation of protein functional class from sparse and imbalanced data sets. In VLDB Workshop on Data Mining and Bioinformatics, pages 65-77. Springer.

Jung, J., Yi, G., Sukno, S. A., and Thon, M. R. (2010). Pogo: Prediction of gene ontology terms for fungal proteins. BMC bioinformatics, 11(1):215.

Kanehisa, M., Goto, S., Kawashima, S., Okuno, Y., and Hattori, M. (2004). The kegg resource for deciphering the genome. Nucleic acids research, 32(suppl_1):D277-D280.

Khan, S., Situ, G., Decker, K., and Schmidt, C. J. (2003). Gofigure: Automated gene ontology ${ }^{\mathrm{TM}}$ annotation. Bioinformatics, 19(18):2484-2485.

Kourmpetis, Y. A., Van Dijk, A. D., Bink, M. C., van Ham, R. C., and ter Braak, C. J. (2010). Bayesian markov random field analysis for protein function prediction based on network data. PloS one, 5(2):e9293.

Kulmanov, M. and Hoehndorf, R. (2020). Deepgoplus: improved protein function prediction from sequence. Bioinformatics, 36(2):422-429.

Kulmanov, M., Khan, M. A., and Hoehndorf, R. (2018). Deepgo: predicting protein functions from sequence and interactions using a deep ontology-aware classifier. Bioinformatics, 34(4):660-668.

Lan, L., Djuric, N., Guo, Y., and Vucetic, S. (2013). Ms-k nn: protein function prediction by integrating multiple data sources. In BMC bioinformatics, volume 14, page S8. Springer.

LeCun, Y., Bengio, Y., and Hinton, G. (2015). Deep learning. nature, 521(7553):436-444.

LeCun, Y., Boser, B. E., Denker, J. S., Henderson, D., Howard, R. E., Hubbard, W. E., and Jackel, L. D. (1990). Handwritten digit recognition with a back-propagation network. In Advances in neural information processing systems, pages 396-404.

Letovsky, S. and Kasif, S. (2003). Predicting protein function from protein/protein interaction data: a probabilistic approach. Bioinformatics, 19(suppl_1):i197-i204.

Li, J., Wang, L., Zhang, X., Liu, B., and Wang, Y. (2020). Gonet: A deep network to annotate proteins via recurrent convolution networks. In 2020 IEEE International Conference on Bioinformatics and Biomedicine (BIBM), pages 29-34. IEEE.

Li, Y., Huang, C., Ding, L., Li, Z., Pan, Y., and Gao, X. (2019). Deep learning in bioinformatics: Introduction, application, and perspective in the big data era. Methods, 166:4-21.

Lobley, A., Swindells, M. B., Orengo, C. A., and Jones, D. T. (2007). Inferring function using patterns of native disorder in proteins. PLoS computational biology, 3(8):e162.

Lobley, A. E., Nugent, T., Orengo, C. A., and Jones, D. T. (2008). Ffpred: an integrated feature-based function prediction server for vertebrate proteomes. Nucleic acids research, 36(suppl_2):W297-W302.

Marcotte, E. M., Pellegrini, M., Thompson, M. J., Yeates, T. O., and Eisenberg, D. (1999). A combined algorithm for genome-wide prediction of protein function. Nature, 402(6757):83-86.

Martin, D. M., Berriman, M., and Barton, G. J. (2004). Gotcha: a new method for prediction of protein function assessed by the annotation of seven genomes. BMC bioinformatics, 5(1):178. 
Min, S., Lee, B., and Yoon, S. (2017). Deep learning in bioinformatics. Briefings in bioinformatics, 18(5):851-869.

Nariai, N., Kolaczyk, E. D., and Kasif, S. (2007). Probabilistic protein function prediction from heterogeneous genome-wide data. Plos one, 2(3):e337.

Nauman, M., Rehman, H. U., Politano, G., and Benso, A. (2019). Beyond homology transfer: Deep learning for automated annotation of proteins. Journal of Grid Computing, 17(2):225-237.

Pandey, G., Kumar, V., and Steinbach, M. (2006). Computational approaches for protein function prediction: A survey. Twin Cities: Department of Computer Science and Engineering, University of Minnesota, 1.

Peng, J., Xue, H., Wei, Z., Tuncali, I., Hao, J., and Shang, X. (2020). Integrating multi-network topology for gene function prediction using deep neural networks. Briefings in bioinformatics.

Pinoli, P., Chicco, D., and Masseroli, M. (2013). Improved biomolecular annotation prediction through weighting scheme methods. In International Meeting on Computational Intelligence Methods for Bioinformatics and Biostatistics, Tenth edition, CIBB 2013, pages 1-12. Università di Salerno.

Pinoli, P., Chicco, D., and Masseroli, M. (2015). Computational algorithms to predict gene ontology annotations. BMC bioinformatics, 16(6):1-15.

Piovesan, D., Giollo, M., Leonardi, E., Ferrari, C., and Tosatto, S. C. (2015). Inga: protein function prediction combining interaction networks, domain assignments and sequence similarity. Nucleic acids research, 43(W1):W134-W140.

Radivojac, P., Clark, W. T., Oron, T. R., Schnoes, A. M., Wittkop, T., Sokolov, A., Graim, K., Funk, C., Verspoor, K., Ben-Hur, A., et al. (2013). A large-scale evaluation of computational protein function prediction. Nature methods, 10(3):221-227.

Rentzsch, R. and Orengo, C. A. (2009). Protein function prediction-the power of multiplicity. Trends in biotechnology, 27(4):210-219.

Rifaioglu, A. S., Doğan, T., Martin, M. J., Cetin-Atalay, R., and Atalay, V. (2019). Deepred: automated protein function prediction with multi-task feed-forward deep neural networks. Scientific reports, 9(1):1-16.

Rost, B., Liu, J., Nair, R., Wrzeszczynski, K. O., and Ofran, Y. (2003). Automatic prediction of protein function. Cellular and Molecular Life Sciences CMLS, 60(12):2637-2650.

Ruepp, A., Zollner, A., Maier, D., Albermann, K., Hani, J., Mokrejs, M., Tetko, I., Güldener, U., Mannhaupt, G., Münsterkötter, M., et al. (2004). The funcat, a functional annotation scheme for systematic classification of proteins from whole genomes. Nucleic acids research, 32(18):5539-5545.

Salakhutdinov, R. and Hinton, G. (2009). Deep boltzmann machines. In Artificial intelligence and statistics, pages 448-455. PMLR.

Sasson, O., Kaplan, N., and Linial, M. (2006). Functional annotation prediction: all for one and one for all. Protein Science, 15(6):1557-1562.

Seyyedsalehi, S. F., Soleymani, M., Rabiee, H. R., and Mofrad, M. R. (2021). Pfp-wgan: Protein function prediction by discovering gene ontology term correlations with generative adversarial networks. Plos one, 16(2): 0244430.

Sharan, R., Ulitsky, I., and Shamir, R. (2007). Network-based prediction of protein function. Molecular systems biology, 3(1):88.

Shehu, A., Barbará, D., and Molloy, K. (2016). A survey of computational methods for protein function prediction. In Big data analytics in genomics, pages 225-298. Springer.

Sleator, R. D. and Walsh, P. (2010). An overview of in silico protein function prediction. Archives of microbiology, 192(3):151-155.

Spalević, S., Veličković, P., Kovačević, J., and Nikolić, M. (2020). Hierachial protein function prediction with tails-gnns. arXiv preprint arXiv:2007.12804.

Stein, L. (2001). Genome annotation: from sequence to biology. Nature reviews genetics, 2(7):493-503.

Szalkai, B. and Grolmusz, V. (2018a). Near perfect protein multi-label classification with deep neural networks. Methods, 132:50-56.

Szalkai, B. and Grolmusz, V. (2018b). Seclaf: a webserver and deep neural network design tool for hierarchical biological sequence classification. Bioinformatics, 34(14):2487-2489.

Szklarczyk, D., Morris, J. H., Cook, H., Kuhn, M., Wyder, S., Simonovic, M., Santos, A., Doncheva, N. T., Roth, A., Bork, P., et al. (2016). The string database in 2017: quality-controlled protein-protein association networks, made broadly accessible. Nucleic acids research, page gkw937. 
Tavanaei, A., Maida, A. S., Kaniymattam, A., and Loganantharaj, R. (2016). Towards recognition of protein function based on its structure using deep convolutional networks. In 2016 IEEE International Conference on Bioinformatics and Biomedicine (BIBM), pages 145-149. IEEE.

Törönen, P., Medlar, A., and Holm, L. (2018). Pannzer2: a rapid functional annotation web server. Nucleic acids research, 46(W1):W84-W88.

Vinayagam, A., del Val, C., Schubert, F., Eils, R., Glatting, K.-H., Suhai, S., and König, R. (2006). Gopet: a tool for automated predictions of gene ontology terms. BMC bioinformatics, 7(1):161.

Wan, C. and Jones, D. T. (2020). Protein function prediction is improved by creating synthetic feature samples with generative adversarial networks. Nature Machine Intelligence, 2(9):540-550.

Watson, J. D., Laskowski, R. A., and Thornton, J. M. (2005). Predicting protein function from sequence and structural data. Current opinion in structural biology, 15(3):275-284.

You, R., Huang, X., and Zhu, S. (2018a). Deeptext2go: Improving large-scale protein function prediction with deep semantic text representation. Methods, 145:82-90.

You, R., Yao, S., Xiong, Y., Huang, X., Sun, F., Mamitsuka, H., and Zhu, S. (2019). Netgo: improving large-scale protein function prediction with massive network information. Nucleic acids research, 47(W1):W379-W387.

You, R., Zhang, Z., Xiong, Y., Sun, F., Mamitsuka, H., and Zhu, S. (2018b). Golabeler: improving sequence-based large-scale protein function prediction by learning to rank. Bioinformatics, 34(14):24652473.

Zdobnov, E. M. and Apweiler, R. (2001). Interproscan-an integration platform for the signaturerecognition methods in interpro. Bioinformatics, 17(9):847-848.

Zehetner, G. (2003). Ontoblast function: From sequence similarities directly to potential functional annotations by ontology terms. Nucleic acids research, 31(13):3799-3803.

Zhang, F., Song, H., Zeng, M., Li, Y., Kurgan, L., and Li, M. (2019). Deepfunc: a deep learning framework for accurate prediction of protein functions from protein sequences and interactions. Proteomics, 19(12):1900019.

Zhang, F., Song, H., Zeng, M., Wu, F.-X., Li, Y., Pan, Y., and Li, M. (2020). A deep learning framework for gene ontology annotation with sequence-and network-based information. IEEE/ACM Transactions on Computational Biology and Bioinformatics.

Zhao, Y., Wang, J., Chen, J., Zhang, X., Guo, M., and Yu, G. (2020). A literature review of gene function prediction by modeling gene ontology. Frontiers in genetics, 11:400.

Zhou, N., Jiang, Y., Bergquist, T. R., Lee, A. J., Kacsoh, B. Z., Crocker, A. W., Lewis, K. A., Georghiou, G., Nguyen, H. N., Hamid, M. N., et al. (2019). The cafa challenge reports improved protein function prediction and new functional annotations for hundreds of genes through experimental screens. Genome biology, 20(1):1-23.

Zou, X., Wang, G., and Yu, G. (2017). Protein function prediction using deep restricted boltzmann machines. BioMed research international, 2017. 